\title{
On Nonexistence of Magnetic Monopoles: Deflection of Magnetic Needle in Static Electric Field of Electric Dipole Implies No Magnetic Field in the Universe
}

\author{
G. H. Jadhav, Member, IACSIT
}

\begin{abstract}
Motion of electrical charged particle in static curled electric field is studied. The electric field exerts asymmetric electric force which forces the particle to follow a curved path. Like in magnetic fields, the path is determined by instantaneous velocity of the particle and the field properties. This way, it shows a resemblance between the asymmetric electric force exerted in static curled electric field and the formal magnetic force exerted in static magnetic field. This resemblance permits to deflect a magnetic needle in static electric field, having non-zero curl, of an electric dipole. It implies the true nature of static magnetic force is static asymmetric electric force and the true nature of static magnetic field is static curled electric field. It provides answer to the question, why the magnetic monopoles are absent in the universe.
\end{abstract}

Index Terms-Electric dipole, magnetic monopoles, Maxwell's equations, static asymmetric electric force, static magnetic force.

\section{INTRODUCTION}

The discovery of magnetic monopoles, elementary particles with net magnetic charge, is one of the most appealing fields of research in theoretical as well as experimental physics. Maxwell's equations formally permit existence of the magnetic monopoles. Dirac, in 1931, has shown the product of basic electric charge and basic magnetic charge is quantized [1]. Immense work on Dirac magnetic monopoles have been published [2]-[13]. Recently, it was found that some condensed matter systems showed a structure superficially similar to magnetic monopoles known as flux tubes. The ends of the tube form a dipole. As the ends can move independently, they can be treated as independent magnetic monopole quasiparticles [14], however, not absolutely like predicted by Dirac. Consequently, no one could report yet about their right subsistence. Literature survey shows that two facts are inadequately understood allied to the subject. One is the behavior of magnetic monopoles in static curled magnetic field and other is the behavior of electrical monopoles in static curled electric field This paper aims to study the later one. For that we produce a static curled electric field theoretically and investigate the motion of an electrical charged particle placed in it. Such

Manuscript received October 31, 2012; revised December 27, 2012. This work is supported by University Grants Commission, New Delhi-110 002, India.

G. H. Jadhav is with the Shri Chhatrapati Shivaji College, Omerga-413606, India (e-mail: ghjadhav@rediffmail.com). type of field, we find, produces asymmetric electric force and forces the particle to follow a curved path. The direction of the path is determined by the instantaneous velocity of the particle in addition to the field properties like in magnetic fields. In this way, the analysis shows a similarity between the static asymmetric electric force and the formal static magnetic force. This similarity allows a magnetic needle to be deflected in a static electric field produced by an electric dipole. This finding helps to understand the mystery of nonexistence of magnetic monopoles.

In Section II, we analyze the motion of an electrical charged particle in a static curled electric field. It confirms the existence of the asymmetric electric force. In Section III, we discuss the resemblance between asymmetric electric force and magnetic force particularly in static fields. In Section IV, we discuss the experimental confirmation of asymmetric electric force. Section V includes a discussion about the results and the last section includes the conclusion.

\section{EXISTENCE OF ASYMMETRIC ELECTRIC FORCE}

For study of asymmetric electric force, we consider an electric field described by the equation

$$
\boldsymbol{E}(r)=\hat{\boldsymbol{x}} \frac{E_{0}}{\sqrt{y^{2}+z^{2}}}=\hat{\boldsymbol{x}} \frac{E_{0}}{r}
$$

This field is parallel to $x$ axis decreasing away from it. Its curl is non-zero. We place a charged particle having net charge $+q$, mass $m$ with initial velocity $v$ in the direction of the field as shown in Fig. 1. The electric field, linked to the particle, is not uniform. It is decreasing in upward direction. We suppose the charged particle is spherical and let the net charge be uniformly distributed on its surface. Therefore, the force applied by the electric field on each unit area on the surface of the sphere is not equal. Hence nature of the force is asymmetric. This asymmetry must reflect into the resultant motion of the particle. To understand this, we divide the sphere of the particle into two hemispheres $A$ and $B$. Further we assume a center of charge on each hemisphere. Suppose $r^{\prime}$ is the distance between these two centers of charges and $r$ is the distance of the center of charge of hemisphere $A$ from $x$-axis (center of the field). Therefore, the net forces in magnitudes on the centers of charge of hemispheres $A$ and $B$, respectively, are

$$
F_{1}=\frac{q E_{0}}{2 r}
$$




$$
F_{2}=\frac{q E_{0}}{2\left(r+r^{\prime}\right)}
$$

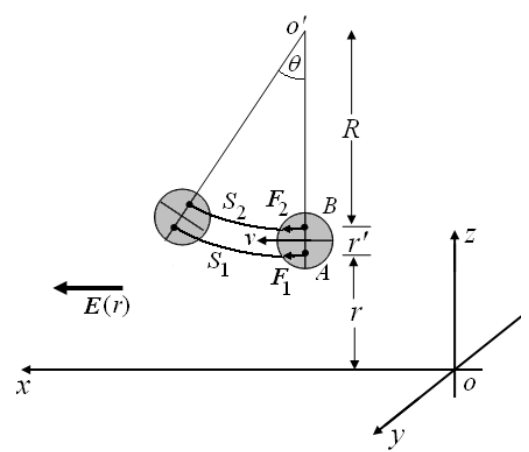

Fig. 1. Charged particle placed in electric field described by equation (1).

Distances $S_{1}$ and $S_{2}$ covered by the centers of charge of hemispheres $A$ and $B$ in small time $t$, respectively, are

$$
\begin{gathered}
S_{1}=v t+\frac{q E_{0}}{2 m} \frac{t^{2}}{r} \\
S_{2}=v t+\frac{q E_{0}}{2 m} \frac{t^{2}}{\left(r+r^{\prime}\right)}
\end{gathered}
$$

Due to the unequal distances $\left(S_{1} \neq S_{2}\right)$ the particle must follow a curved path turning into the weak field as shown in the figure.

The radius $R$ of the curve is

$$
R=\frac{r^{\prime} S_{2}}{S_{1}-S_{2}}=r\left(\frac{2 m v}{q E_{0} t}\left(r+r^{\prime}\right)+1\right)
$$

It is the radius of the curved path started by the particle at initial point where its velocity was parallel to the electric field or to the force.

As $r^{\prime} \rightarrow 0$, the particle size reduces to zero, equation (6) gives

$$
R=r\left(\frac{2 m v r}{q E_{0} t}+1\right)
$$

Thus, though the particle size becomes zero, the radius of the curved path followed by the particle cannot be infinite or the path cannot be a straight line. Therefore, any electrical charged particle, whatever may be the size, must follow a curved path subjected to the electric field described by equation (1).

For zero initial velocity,

$$
R=r
$$

The curved path unexpectedly becomes independent of all parameters except $r$, which is the distance of the particle from $x$-axis (center of the field).

The particle having initial velocity in opposite direction of the field gets decelerated resulting into $S_{1}<S_{2}$. In this case the radius of the path obeys the equation

$$
R=\left(\frac{2 m r v}{q E_{0} t}-1\right)\left(r+r^{\prime}\right)
$$

Here the particle turns into the stronger field as shown by path (b) in Fig. 2. If the particle tries to travel in the direction perpendicular to the field but towards the weak field then gets turned in opposite direction of the force (path (c)). In this case, the center of charge of hemisphere $A$ moves faster than the center of charge of hemisphere $B$ in the direction of the force which makes the particle to move in the opposite direction of the force. And if the particle tries to travel in the direction perpendicular to the force but towards the strong field then it gets turned along the direction of the force (path (d)). Thus, the initial velocity of the particle and the field properties, such as the direction of the field and in which direction it is varying, mutually determine the direction along which the particle to be turned. This property is similar to the property of magnetic field. Such similarity was also discussed in [15] with introducing a new concept called 'force through field-field interaction'. However, the present treatment uses the usual electric force equation, $\boldsymbol{F}=q \boldsymbol{E}$, therefore, it becomes significant.

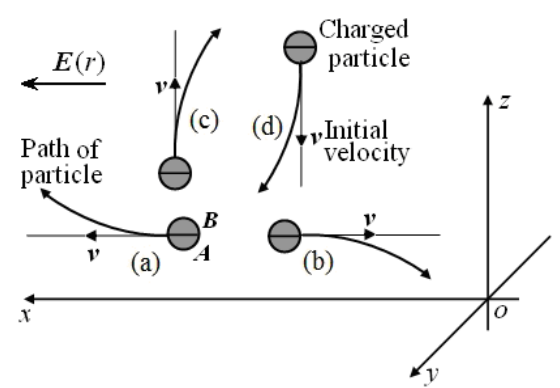

Fig. 2. Charged particles with different initial velocities in the parallel electric field described by equation (1).

\section{RESEMBLANCE BETWEEN STATIC ASYMMETRIC ELECTRIC FORCE AND STATIC MAGNETIC FORCE}

By analyzing motions of the charged particle in Fig. 2, we realize that we are already acquainted with such type of motions. These are the motions of electrical charged particles in the magnetic field of a straight long conductor carrying a steady electric current as shown in Fig. 3.

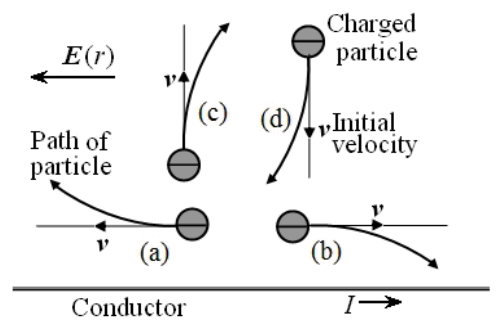

Fig. 3. Charged particles with different initial velocities in the field of a straight long conductor carrying a steady electric current.

Thus, a question arises, whether the current carrying conductors produce magnetic fields or really they produce parallel electric fields. For instance, if one supposes the conductors are producing parallel electric fields in opposite direction of the current then the electric field produced by the conductor in Fig. 3 may be represented by the equation 


$$
\boldsymbol{E}(r)=-k \frac{\boldsymbol{I}}{r}
$$

where $k$ is a proportionality constant and $r$ is the perpendicular distance of the field point from the conductor.

In fact, two different things (electric field and magnetic field) cannot produce the same event (same deflection of the charged particle) independently. Therefore, the current carrying conductor must be either producing a parallel electric field or producing a circular magnetic field. In fact, if both fields exist, may be at different places, and produce work in the same fashion then it will be a wonder. Therefore, we suppose the conductor carrying the steady electric current is really producing a parallel electric field given by equation (10) then a current carrying circular coil and hence a bar magnet must produce circular electric fields around themselves. Therefore, the deflections of a charged particle observed theoretically in circular electric fields and the deflection of the same charged particle observed experimentally in the fields of magnetic bars must be same. To verify that we analyze theoretically deflection of a charged particle in a circular electric field and compare it with the experimental observations. However, one has to consider two types of circular electric fields; one's intensity is decreasing and the other's intensity is increasing while going away from their centers.

\section{A. Electric Field Decreasing Away from Center}

First we consider the circular electric field whose intensity is decreasing away from its center. The cross section of the field is shown in Fig. 4. The field intensity is constant along the line perpendicular to the paper. If any positively charged particle tries to move towards the center of the field, it should get deflected as shown in the figure. It can be understood with the help of Fig. 2.

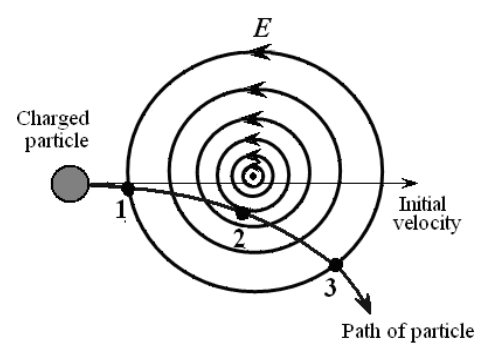

Fig. 4. Motion of charged particle in circular electric field whose intensity is decreasing away from the center.

\section{1) Comparison with experimental observations}

For experimental observations, we have to guess a plausible electric field to a bar magnet. In a bar magnet when we look from its $\mathrm{S}$ pole to $\mathrm{N}$ pole, the net spin current constituted by the unpaired electrons is in clockwise direction. Therefore, the plausible electric field must be in opposite direction of the spin current. Further this electric field, in the region between the poles, should decrease while going away from the magnet in the direction perpendicular to the axis (a straight line passing through the two poles) of the magnet. Hence, when we look from $\mathrm{S}$ pole to $\mathrm{N}$ pole of the bar magnet the appeared electric field must be looked like the electric field as shown in Fig. 4. Therefore, when a charged particle approaches such a bar magnet from its left side, as shown in Fig. 5, should be deflected in downward direction. No doubt, it is experimentally observed.

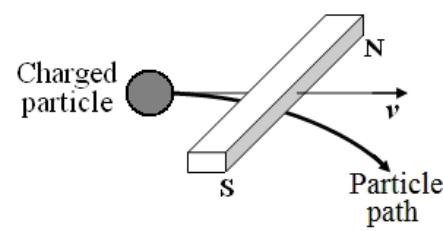

Fig. 5. Charged particle in the field of bar magnet between the two poles.

\section{B. Electric Field Increasing Away from Center}

Second we consider the circular electric field whose intensity is increasing while going away from its center as shown in Fig. 6. In this case the particle motion gets inverted relative to the motion observed in the first case. We note that the direction of the field is not changed. But there is a difference in the change in intensity which inverted the motion of the particle. It may be understood by Fig. 2 .

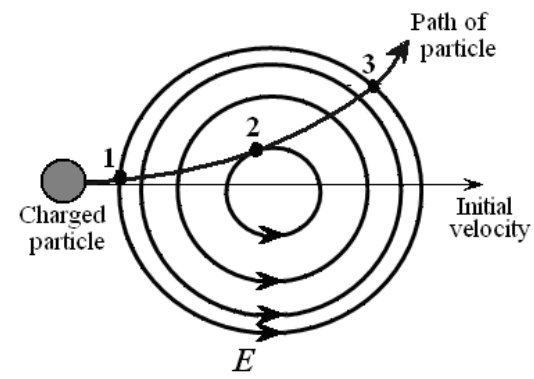

Fig. 6. Motion of charged particle in circular electric field whose intensity is increasing away from the center.

\section{1) Comparison with experimental observations}

Experimentally it can also be observed when the particle is shifted at any one end of the bar magnet as shown in Fig. 7. The deflection gets inverted as like observed in Fig. 6 indicating that the direction of the electric field in this region is not changed as there is no change in the spin current but the field must increase away from the axis of the bar magnet. Further experimental observations show that the electric field increases until the surface of the cone where, in general, we assume the formal magnetic field lines are used to turn in opposite direction. After the surface of the cone, the circular electric field again decreases in perpendicular direction to the axis of the magnet.

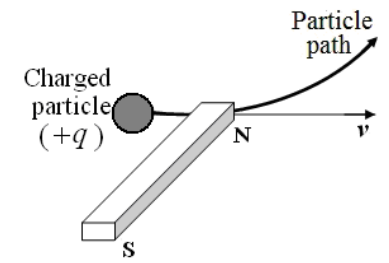

Fig. 7. Charged particle in the field of bar magnet in the region after its one end.

\section{Attraction and Repulsion between Poles of Bar}

Magnets on the Basis of Circular Electric Field

If the circular electric field of a bar magnet is responsible to produce the deflection of the charged particle then this field must be responsible to produce the attraction and 
repulsion between the poles of the bar magnets. It can be demonstrated as follows.

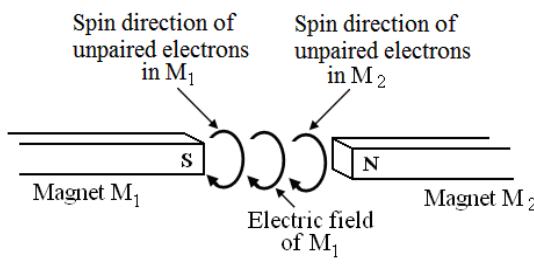

Fig. 8. Representation of attraction between magnetic poles in terms of asymmetric electric force and circular electric field.

Let us verify the attraction between two magnetic poles of two different bar magnets. Fig. 8 illustrates the direction of the electric field and spin motion of unpaired electrons of bar magnet $M_{1}$. The unpaired electrons in bar magnet $M_{2}$ are spinning in the same direction of the electric field of magnet $M_{1}$. Therefore, the force imposed by the field of magnet $M_{1}$ should try to decelerate the spin velocity of the unpaired electrons of magnet $\mathrm{M}_{2}$. Because of this attempt the unpaired electrons in magnet $\mathrm{M}_{2}$ should be pulled towards the end of magnet $\mathrm{M}_{1}$ as the electric field near the end is strong. In the same way the magnet $M_{2}$ should react with magnet $M_{1}$. Therefore, both ends should get dragged towards each other. When the electric field having non-zero curl tries to decelerate a charged particle, the particle gets dragged towards the strong field. This process can be understood from Fig. 2. Further when like poles are close to each other, the electric field of one bar magnet should be in attempt to accelerate the spin velocity of the unpaired electrons of the other bar magnet. Because of this attempt the unpaired electrons of each bar magnet should get pushed away from the other bar magnet. Therefore, they should be able to produce the illusion of repulsion. In this way we have seen that, though there is no any actual direct force of attraction or repulsion between the poles or ends of bar magnets, a torque, produced because of the circular asymmetric electric force, is responsible to produce such illusion. For paired electrons the net effect of asymmetric electric force is null.

\section{EXPERIMENT TO VERIFY EXISTENCE OF ASYMMETRIC ELECTRIC FORCE}

Experimental verification of asymmetric electric force requires a static electric field either parallel or circular having non-zero curl. Such type of field may be produced by an electric dipole (Fig. 9). The field at point $p$ is

$$
\boldsymbol{E}=\frac{1}{4 \pi \varepsilon_{0}} \frac{\hat{z} 2 q a}{\left(a^{2}+y^{2}\right)^{3 / 2}}
$$

where $\hat{z}$ is unit vector along $\mathrm{Z}$-axis.

Curl of this field is

$$
\nabla \times \boldsymbol{E}=\frac{-1}{4 \pi \varepsilon_{0}} \frac{\hat{\boldsymbol{x}} 6 a q y}{\left(a^{2}+y^{2}\right)^{5 / 2}} \neq 0
$$

where $\hat{\boldsymbol{x}}$ is unit vector along $x$-axis.
The curl of the field is non zero, therefore, it must produce an asymmetric electric force on a charged particle. But this non-zero curl value produces a paradox. Third Maxwell's equation is

$$
\nabla \times \boldsymbol{E}=-\frac{\partial \boldsymbol{B}}{\partial t}
$$

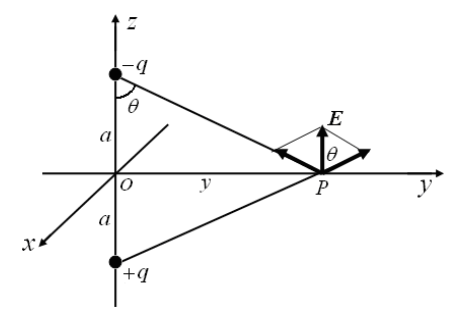

Fig. 9. Electric field of an electric dipole.

For the above electric dipole, it gives

$$
\boldsymbol{B}=\left(\frac{1}{4 \pi \varepsilon_{0}} \frac{\hat{\boldsymbol{x}} 6 a q y}{\left(a^{2}+y^{2}\right)^{5 / 2}}\right) t
$$

Thus, the electric dipole should produce a circular magnetic field (Fig. 10) which should increase with time. This is inconsistent with the experimental observations. Nowhere it is mentioned yet.

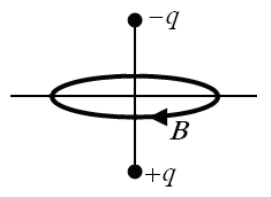

Fig. 10. Expected magnetic field of an electric dipole according to the third Maxwell's equation.

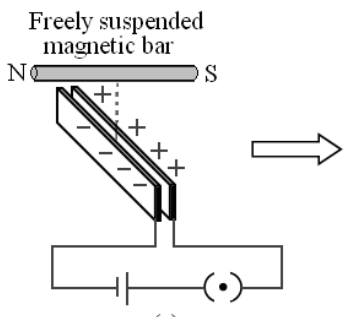

(a)

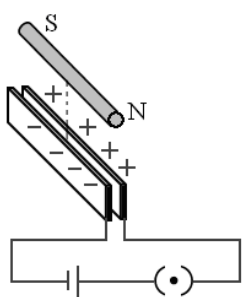

(b)
Fig. 11. Rotation of bar magnetic (magnetic needle) in the field of electrically charged plates.

For experimental confirmation of the asymmetric electric force, the electric field described by equation (11) can be produced experimentally by charging two parallel metallic plates separated by a small distance as shown in Fig. 11 (a). We may suspend a small magnetic bar, free to rotate, above the plates. The system should be enclosed in a vacuum glass chamber so as to form a proper electric field between the plates and to have no electric current in the region between the plates. The plates should be kept parallel to east-west direction. Before charging the plates the magnet will be well aligned in south-north direction. Now charging the plates should cause to rotate the magnetic needle and finally get aligned along the length of the plates as indicated in Fig. 11 (b). It may require sufficient charge on the plates. By 
reversing the charge on the plates the magnetic bar should be aligned to the plates in opposite direction. This should happen because of the existence of asymmetric electric force on the charged particles, especially on the unpaired electrons, in the bar magnet. Explanation for the expected result is cited below.

The electric field of the plates in the region of the magnetic bar is directed from right to left applying force on the electrons from left to right. The magnitude of the force is decreasing in upward direction. The unpaired electrons of the magnetic bar, while looking from $\mathrm{N}$ pole to $\mathrm{S}$ pole, are spinning in clockwise direction. Therefore, in Fig. 11 (b), the unpaired electrons must be experiencing net decelerating force. Since, at any instant the decelerating force on the lower hemisphere of any such electron is greater than the accelerating force on its upper hemisphere. Therefore, such electrons and hence the bar magnet too should experience a downward pull towards the plates. Since for net decelerating force the particle gets dragged into strong field as illustrated by curve (b) in Fig. 2. For paired electrons, this effect is zero. Obviously, the downward pull will be stronger when the bar will get perfectly aligned to the plates as shown in Fig. 11 (b). This is the reason why the bar magnet should get finally aligned to the plates whatever may be its initial angle with respect to the plates. By reversing the charge on the plates, the bar should get aligned in opposite direction.

\section{DISCUSSION}

In section IV, we have found that the formal classical electrodynamics allows a magnetic needle to be deflected in static electric field of an electric dipole. This is inconsistent with the concept of magnetic field, since, in 1820 Oersted first time noted a deflection of magnetic needle in the vicinity of a conductor carrying electric current which leaded to the conclusion that the conductor was producing a magnetic field. That means magnetic needle could be deflected in magnetic fields only. Consequently the deflection of magnetic needle in static electric field of an electrical dipole is inconsistent with the previous conviction. Further, we understood that third Maxwell's equation forces a static electric dipole to produce a magnetic field around itself which increases with time. This is inconsistent with the experimental observations too. The classical theory of electrodynamics thus contradicts itself. Further, we don't find the existence of any magnetic monopoles. This happened because of improper understanding of the electric force and hence the electric field In fact, in absence of magnetic monopoles, why the electric charges should produce magnetic field is a question. The present investigation is made to find out the answer of this question, which brought us to the conclusion that the true nature of magnetic force is the asymmetric electric force and the real nature of static magnetic field is the static curled electric field. At first, we have shown that a straight long conductor, carrying steady electric current, produces a parallel electric field instead of the circular magnetic field. Consequentially a current carrying circular coil and hence a bar magnet must produce a circular electric field around themselves. No doubt these fields have non-zero curl responsible to produce the asymmetric electric force. Therefore, one should always divide the whole electric field into divergent electric field and curled electric field. In the divergent electric field the electric force is symmetric which forces the subjected particle to follow a straight path. In the curled electric field, may be parallel or circular, the electric force is asymmetric which forces the subjected particle to follow a curved path. Due to lack of this knowledge we were considering the curled part of the electric field as magnetic field and expecting the magnetic monopoles to be materialized which we couldn't. Another explored important fact is that we assume a direct force of attraction and repulsion between the poles or ends of bar magnets. But it is found that this conviction is wrong. There is no any such direct force of attraction or repulsion but a circular asymmetric electric force or a torque is responsible to produce such effect. The unnecessary entry of magnetic field kept us unaware of this fact.

\section{CONCLUSION}

There is no any magnetic field existing in the universe. The true nature of the supposed magnetic force is asymmetric electric force and the true nature of the supposed magnetic field is that part of electric field which has non-zero curl. There is no any direct force of attraction and repulsion between the poles or ends of bar magnets. A circular asymmetric electric force is responsible to produce such illusion. There is no any reason to exist magnetic monopoles in the universe.

\section{ACKNOWLEDGMENT}

The author is thankful to the University Grants Commission, New Delhi, India for financial support to this work.

\section{REFERENCES}

[1] P. A. M. Dirac, "Quantized singularities in the electromagnetic field," Proc. Roy. Soc. Lond., 1931, vol. 133, pp. 60-72.

[2] J. Schwinger, "Magnetic charge and quantum field theory," Phys. Rev., 1966, vol. 144, pp. 1087-1093.

[3] T. T. Wu and C. N. Yang, "Dirac monopole without strings: monopole harmonics," Nucl. Phys., 1976, vol. B 107, pp. 365-380.

[4] Y. Kazama, C. N. Yang, and A. S. Goldhaber, "Scattering of a Dirac particle with charge Ze by a fixed magnetic Monopole," Phys. Rev., 1977, vol. D 15, pp. 2287-2299.

[5] G. T. Hooft, "Magnetic monopoles in unified gauge theories," Nucl. Phys., 1974, vol. B 29, pp. 276-284.

[6] A. M. Polyakov, "Particle spectra in quantum field theory," JETP Lett., 1974, vol. 20, pp. 194-195.

[7] S. W. Barwick, K. Kinoshita, and P. B. Price, "Search for penetrating, highly charged particles at mountain altitude," Phys. Rev., 1983, vol. D 28, pp. 2338-2340.

[8] P. Musset and M. Price, "Search for magnetic monopoles in electron-positron collisions at 34 Gev C.M. energy," Phys. Lett., 1983 , vol. B 128 , pp. 333-335

[9] M. Bertani, G. Giacomelli, M. R. Mondardini, B. Pal, L. Patrizii, F. Predieri, P. Serra-Lugaresi, G. Sini1, M. Spurio, V. Togo, and S Zucchelli, "Search for magnetic monopoles at the Tevatron Collider," Europhys. Lett., 1990, vol. 12, pp. 613-616.

[10] R. D. Gardner, B. Cabrera, M. E. Huber, and M. A. Taber, "Search for cosmic ray magnetic monopoles using a three-loop superconductive detector," Phys. Rev., 1991, vol. D 44, pp. 622-635.

[11] J. L. Thron, W. W. M. Allison, G. J. Alner, I. Ambats, D. S. Ayres, and 
L. J. Balka, "A search for magnetic monopoles with the SOUDAN-2 detector," Phys. Rev., 1992, vol. D 46, pp. 4846-4851.

[12] Y. D. He, "Search for a Dirac magnetic monopole in high energy nucleus nucleus collisions," Phys. Rev. Lett., 1997, vol. 79, pp 3134-3137.

[13] P. Irwin, "SU(3) monopoles and their fields," Phys. Rev., 1997, vol. D 56, pp. 5200-5208.

[14] C. Castelnovo, R. Moessner, and S. L. Sondhi, "Magnetic monopoles in spin ice," Nature, 2008, vol. 451, pp. 42-45.

[15] G. H. Jadhav, "On true face of magnetic field," Adv. Mater. Res., 2012, vol. 433-440, pp. 272-280.
G. H. Jadhav was born on March 05,1964 , in Omerga, Inda. He got B.Sc. in physics, electronics and mathematics in 1987 and M.Sc. in Physics in 1989 from Marathwada University, Aurangabad, India. He got Ph.D. in theoretical physics in Feb, 2002 from Dr. Babasaheb Ambedkar Marathwada University, Aurangabad, India. Currently he is working on a major research project entitled "Reinvestigation of magnetic field on absence of magnetic monopoles in the universe" sanctioned by the University Grants Commission, New Delhi, India. His fields of research interest are condensed matter physics and theoretical physics. His scientific works had been published at many reputable international journals proceedings, and international conferences. 